\title{
The Inhibitory Effect of Cranberry Juice on Phenytoin Metabolism by Human Liver Microsomes
}

\author{
Kentarou USHIJIMA $^{* 1}$ Taka-aki KOSHIMIZU*2 ${ }^{* 2}$ Akio FUJIMURA*1 \\ *1 Divisions of Clinical Pharmacology, Department of Pharmacology, Jichi Medical University, Tochigi, Japan \\ *2 Divisions of Molecular Pharmacology, Department of Pharmacology, Jichi Medical University, Tochigi, Japan
}

\begin{abstract}
CYP2C9 is involved in the metabolism of many drugs such as warfarin and phenytoin (PHT). It is well known that some foods cause pharmacokinetic alterations of drugs and lead to toxicity. Regarding the interaction caused by cranberry juice $(\mathrm{CrJ})$, its influence on the metabolism of warfarin, a CYP2C9 substrate, is controversial. In addition, information concerning the influence of $\mathrm{CrJ}$ on the pharmacokinetics of other substrate drugs of CYP2C9 is not available. To examine the inhibitory effect of CrJ on the metabolism of PHT, we performed experiments using a baculovirus expression microsome system and human liver microsome assay. CrJ concentration-dependently inhibited the substrate metabolism both in a baculovirus-expressed microsome system and in human liver microsome assay. These data support the idea that CrJ inhibits CYP2C9 activity, and the subsequent metabolism of drugs metabolized by CYP2C9. In this study, we did not identify the specific substance(s) which inhibits CYP2C9 activity. However, as CrJ did not enhance the PHT accumulation in 3DHepG2 cells, it is thought that the penetration into hepatic cells of inhibitory substances in CrJ seems to be small.
\end{abstract}

Key words : cranberry juice, CYP2C9, human liver microsomes, interaction, phenytoin

\section{Introduction}

CYP2C9 is involved in the metabolism of many drugs including (S)-warfarin, tolbutamide, nonsteroidal anti-inflammatory drugs and phenytoin $(\mathrm{PHT})^{1,2)}$. Some drugs with a narrow therapeutic range such as $(\mathrm{S})$-warfarin and PHT are particularly critical because even small pharmacokinetic and/or pharmacodynamic alterations can lead to toxicity or ineffectiveness. Regarding this issue, the interaction of foods and beverages with drugs should be taken into consideration in clinical practice. Recently, several case reports showed that patients on warfarin therapy suffered from a profound hypoprothrombinemia after the ingestion of cranberry juice $(\mathrm{CrJ})^{3,4)}$. These data led us to speculate that $\mathrm{CrJ}$ inhibits CYP2C9 activity. On the other hand, there are reports indicating that $\mathrm{CrJ}$ has a minimal effect on the pharmacokinetics and pharmacodynamics of warfarin $^{5,6)}$. However, we think that it is too early to reach a conclusion about the effect of $\mathrm{CrJ}$ on CYP2C9 activity, because, although $\mathrm{CrJ}$ is reported to inhibit metabolism of CYP2C9 substrates ${ }^{7}$, such effect of $\mathrm{CrJ}$ has not been sufficiently evaluated in vitro, and thus there are controversial results between in vivo and in vitro findings.

To address the issue, we examined the effect of $\mathrm{CrJ}$ on the metabolism of PHT, another CYP2C9 substrate $^{8)}$. In this study, we determined the inhibitory effect of CrJ on CYP2C9 activity using an available commercialized inhibitor-screening kit. We also examined the influence of $\mathrm{CrJ}$ on the PHT metabolism by human liver microsomes.

\section{Materials and Methods}

\section{Materials}

The beverage used in this study was cranberry juice (containing 27\% cranberry; Ocean Spray Cranberry, Inc., Lakeville-Middleboro, MA). Pooled human liver microsomes (from 15 donors) and CYP2C9 inhibitor screening kit were obtained from BD Bioscience (Franklin Lakes, NJ). Sodium Phenytoin $(5 \mathrm{w} / \mathrm{v} \%)$ was purchased from Dainippon Sumitomo Pharma Co., Ltd. (Osaka, Japan). Sulfaphenazole (SFZ), fluvoxamine maleate, rifampicin and Dulbecco's Modified Eagle Medium (DMEM) were

Address for correspondence : Fujimura A. Divisions of Clinical Pharmacology, Department of Pharmacology, Jichi Medical University, 3311-1, Yakushiji, Shimotsuke, Tochigi 329-0498, Japan

(Manuscript received July 28, 2008 ; revised November 20, 2008 ; accepted December 17, 2008) 
obtained from Sigma-Aldrich (St. Louis, MO).

\section{Extraction of beverage with ethyl acetate}

Preparation of the cranberry juice (CrJ) extract was performed according to the method reported previously ${ }^{9}$. CrJ was mixed with 3 -fold volumes of ethyl acetate and shaken vigorously for $20 \mathrm{~min}$, and the mixture was centrifuged at $900 \mathrm{~g}$ for $10 \mathrm{~min}$. The aqueous phase was discarded and the organic layer was evaporated to dryness under a stream of nitrogen. The residue was reconstituted with methanol (or acetonitrile in MFC-kit experiment according to the recommendation in the instruction manual) of one-fifth the initial respective volume of juice. The reconstituted solution was designated as a 5-fold concentrate $(500 \%$ extract $)$.

\section{Effect of CrJ extracts on the metabolism of CYP2C9 substrate}

The inhibitory effect of $\mathrm{CrJ}$ extract and SFZ on CYP2C9 activity was determined by baculovirus expression system using fluorescence substrate (MFC : 7-methoxy-4-trifluoro-methylcoumarin) (BD Bioscience). The experiment was performed according to the instruction manual ; $100 \mu \mathrm{L}$ of the reaction mixture contained NADP, glucose 6-phosphate, glucose-6-phosphate dehydrogenase and $\mathrm{MgCl}_{2}$ with appropriate concentrations of $\mathrm{CrJ}$ extract (final extract concentration; $0.0046-10 \%$ ) or SFZ (final concentration ; $0.005-10 \mu \mathrm{M})$, and was pre-incubated for $10 \mathrm{~min}$ at $37^{\circ} \mathrm{C}$. The second reaction was started by the addition of $100 \mu \mathrm{L}$ of the substrate mixture containing $\mathrm{MFC}$ (final concentration ; $75 \mu \mathrm{M}$ ) and cDNA-expressed CYP2C9, P450 reductase and cytochrome b5 using a baculovirus expression system in insect cell microsomes, and incubated for $45 \mathrm{~min}$ at $37^{\circ} \mathrm{C}$. The reaction was stopped by the addition of 75 $\mu \mathrm{L}$ of $0.5 \mathrm{M}$ Tris/acetonitrile $(1: 4)$. The values of fluorescence of HFC (7-hydroxytrifluoromethylcoumarin, metabolite of MFC) were measured with the wavelength at $405 \mathrm{~nm}$ (excitation) and $530 \mathrm{~nm}$ (emission). Concentration-inhibition relationships were fitted to a four-parameter logistic equation using a non-linear curve-fitting program (Igor Pro 6.03, WaveMetrics, Lake Oswego, OR), from which the $\mathrm{IC}_{50}$ values were derived ${ }^{10)}$. During an iterative curve fitting, no restriction was set for all values of coefficients. The intra- and inter-assay coefficients of variation were better than $5 \%$.

\section{Influence of $\mathrm{CrJ}$ on the metabolism of PHT by human liver microsomes}

Human liver microsome assay was performed according to the previous report with a minor modification $^{9)}$. In brief, $280 \mu \mathrm{L}$ of reaction mixture was pre-incubated for $10 \mathrm{~min}$ at $37^{\circ} \mathrm{C}$ by addition of human liver microsomes (final concentration ; $0.48 \mathrm{mg} / \mathrm{mL}$ ) to the NADPH-regenerating system (1.3 mM NADP, $3.3 \mathrm{mM}$ glucose 6 -phosphate, $0.4 \mathrm{U} / \mathrm{mL}$ glucose-6phosphate dehydrogenase, $3.3 \mathrm{mM} \mathrm{MgCl}_{2}$ ) in $50 \mathrm{mM}$ phosphate buffer (pH7.4) with $10 \mu \mathrm{L}$ of $\mathrm{CrJ}$ extract (final extract concentration; 0.3 and $1 \%$ ) or SFZ solution (final concentration; 0.3 and $1.0 \mu \mathrm{M}$ ). After pre-incubation, $10 \mu \mathrm{L}$ of PHT solution was added (final concentration; $10 \mu \mathrm{g} / \mathrm{mL}$ ) and the mixture was incubated for $40 \mathrm{~min}$ at $37^{\circ} \mathrm{C}$, because the rate of reduction of $\mathrm{PHT}$ remained constant for up to $40 \mathrm{~min}$ under these conditions.

The reaction was stopped by the addition of $1.2 \mathrm{~mL}$ of dichloromethane to the $300 \mu \mathrm{L}$ of reaction mixture. Sample was shaken for 5 min vigorously and centrifuged at $750 \mathrm{~g}$ for $2 \mathrm{~min}$. The aliquot layer was discarded and the organic solvent $(900 \mu \mathrm{L})$ was evaporated to dryness under a stream of nitrogen. The residue was dissolved in $120 \mu \mathrm{L}$ of $50 \mathrm{mM} \mathrm{NaOH}$ and PHT concentration in the solution was automatically measured by fluorescence polarization immunoassay (FPIA) method (TDx ${ }^{\circledR}$, Abbott Japan, Co., Ltd., Tokyo, Japan). The intra- and inter-assay coefficients of variation, determined by 5 and $20 \mu \mathrm{g} / \mathrm{mL}$ of PHT were better than $5 \%$.

\section{Analysis of the inhibition pattern of $\mathrm{CrJ}$ on the PHT metabolism}

To investigate the inhibition pattern of $\mathrm{CrJ}$ on $\mathrm{PHT}$ metabolism, Lineweaver-Burk plot analysis was performed. In brief, the reaction mixture contained human liver microsomes, the NADPH-regenerating system, CrJ extract (final extract concentration; $0.3 \%$ and $1 \%$ ) was pre-incubated. After pre-incubation, PHT solution was added (final concentration ; 4, 10 and $20 \mu \mathrm{g} / \mathrm{mL}$ ) and the mixture was incubated for 40 min. After stopping the reaction, the applied procedures were the same as described above.

\section{Influence of fluvoxamine on the metabolism of PHT by human liver microsomes}

To investigate the suppression on the PHT metabolism caused by the CrJ-mediated inhibition of 
CYP2C9, fluvoxamine was applied to this human liver microsome assay. As described above, the reaction mixture contained human liver microsomes, the NADPH-regenerating system, CrJ extract (final extract concentration ; 1\%), and fluvoxamine (final extract concentration; 1 and $10 \mu \mathrm{M}$ ) was preincubated. After pre-incubation, PHT solution was added (final concentration ; $10 \mu \mathrm{g} / \mathrm{mL}$ ) and the mixture was incubated for $40 \mathrm{~min}$. After stopping the reaction, the applied procedures were the same as described above.

\section{Influence of $\mathrm{CrJ}$ on the PHT accumulation in 3D-HepG2 cell}

HepG2 cells in 3-dimension matrix (3D-HepG2) were purchased from Applied Cell Biotechnologies, Inc. (Kanagawa, Japan) and were cultured in DMEM with $10 \%$ fetal bovine serum (FBS) in a 24 -well plate at $37^{\circ} \mathrm{C}$ with $5 \% \mathrm{CO}_{2}$. Cells were exposed to $20 \mu \mathrm{M}$ rifampicin for $48 \mathrm{hr}$ to induce CYP enzymes. Thereafter, media were replaced with FBS-free DMEM containing $1 \mu \mathrm{M} \mathrm{SFZ}$ or $1 \% \mathrm{CrJ}$ and cells were incubated for $1 \mathrm{hr}$. After pre-treatment, PHT solution (final concentration; $10 \mu \mathrm{g} / \mathrm{mL}$ ) was added to medium and cells were incubated for $40 \mathrm{~min}$. Medium was collected and the reaction was stopped by washing with phosphate-buffered saline, followed by treating with $150 \mu \mathrm{L}$ of $0.05 \mathrm{~N} \mathrm{NaOH} / 0.1 \%$ Triton X-100 solution. The 3-dimension matrixes were incubated for $2 \mathrm{hr}$ at room temperature to digest cells for measurement of PHT and protein concentration. PHT concentration was measured by $\mathrm{TDx}^{\circledR}$, and protein concentration was assayed by BCA protein assay reagent kit (Thermo Fisher Scientific Inc., Rockford, IL). PHT concentrations in 3D-HepG2 cells were calculated by the adjustment per mg protein.

\section{Statistical analysis}

Groups were compared by one-way ANOVA, followed by Tukey-Kramer's test. $p<0.05$ was considered to be significant.

\section{Results}

\section{Inhibitory effect of $\mathrm{CrJ}$ extract on the $\mathrm{HFC}$ production}

To determine the inhibitory effect of $\mathrm{CrJ}$ extract on human CYP2C9 activity, we used a commercialized kit using a baculovirus expression system. SFZ, a positive control, concentration-dependently inhibited
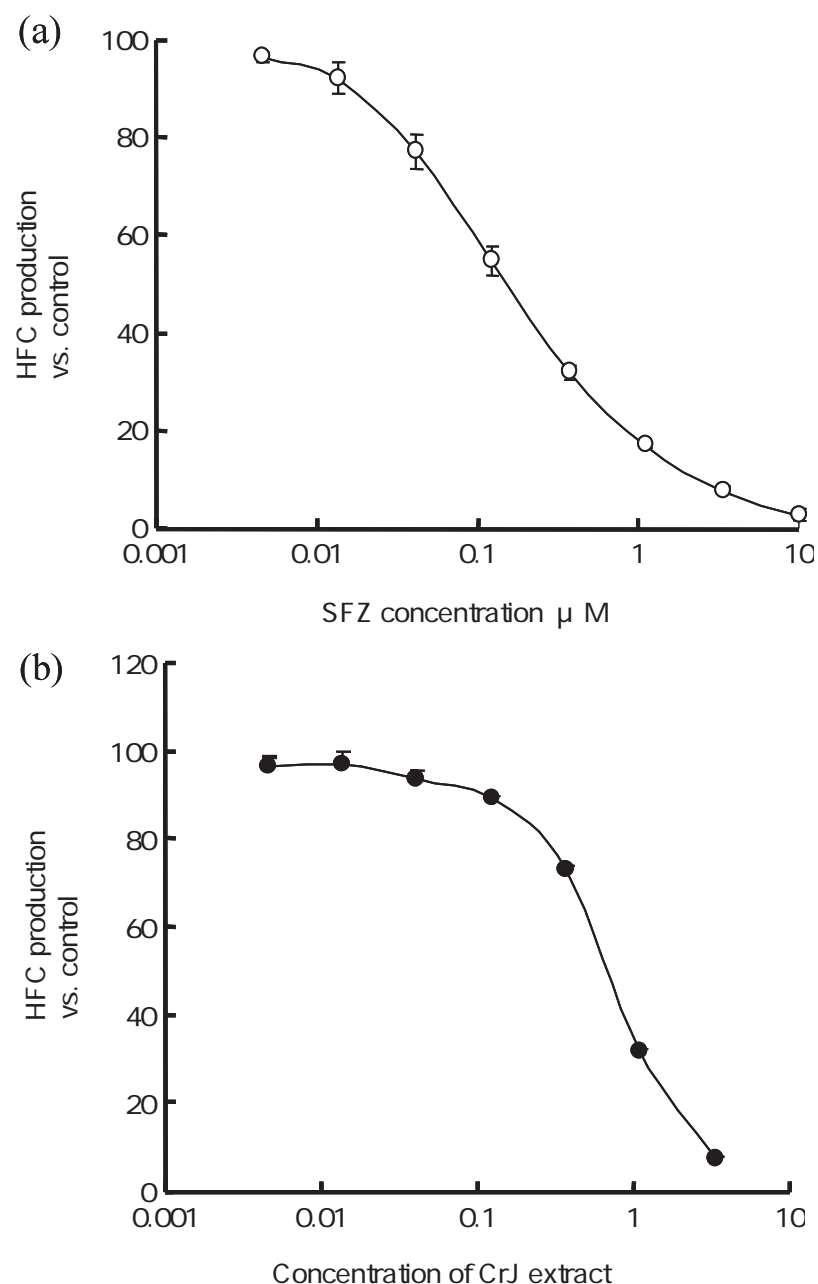

Fig. 1 Effect of SFZ (a) and CrJ extract（b) on the metabolism of MFC

The $\mathrm{IC}_{50}$ values were calculated to be $0.15 \mu \mathrm{M}$ (SFZ) and $0.74 \%(\mathrm{CrJ})$. CrJ concentration indicates the original concentration of $\mathrm{CrJ}$. Mean \pm S.D. $\mathrm{n}=3$.

the $\mathrm{HFC}$ production, and the $\mathrm{IC}_{50}$ value was calculated to be $0.15 \mu \mathrm{M}$ (Fig. 1a). As shown in Fig. 1b, CrJ extract also showed concentration-dependent inhibition on the $\mathrm{HFC}$ production and the $\mathrm{IC}_{50}$ value was $0.74 \%$.

\section{Influence of $\mathrm{CrJ}$ extracts on the metabolism of PHT by human liver microsomes}

To examine whether the extract of $\mathrm{CrJ}$ inhibited the PHT metabolism, we performed an experiment using human liver microsome with NADPHregeneration system. In this assay, we measured the parent drug (PHT), and calculated the reduction of PHT concentration, which was considered to represent metabolism of PHT. SFZ significantly inhibited 
(a)

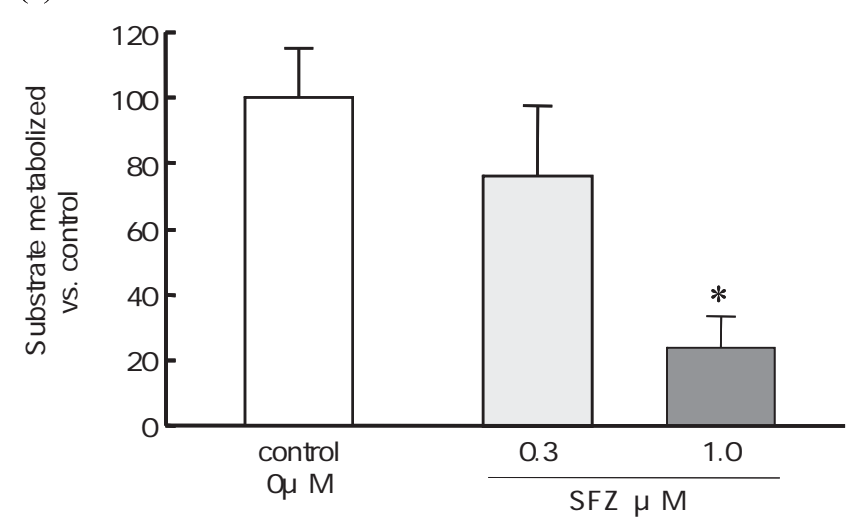

(b)

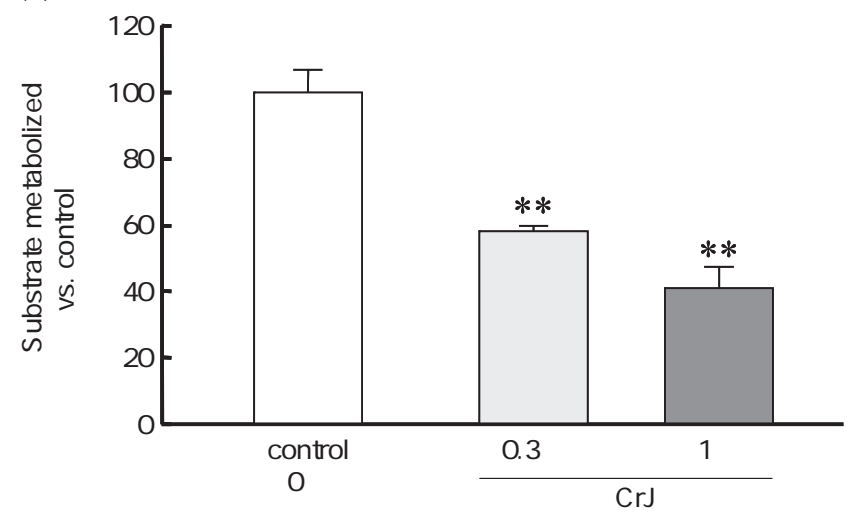

Fig. 2 Effect of SFZ (a) and CrJ extract (b) on the metabolism of PHT by human liver microsomes

Initial concentration of PHT was $10 \mu \mathrm{g} / \mathrm{mL}$ and the incubation time was $40 \mathrm{~min}$. CrJ concentration indicates the original concentration of $\mathrm{CrJ} .{ }^{*}, p<0.05 ;{ }^{* *}, p<0.01$ compared with control $(0 \mu \mathrm{M}$ or $0 \%)$. Mean \pm S.D. $\mathrm{n}=3$.

the PHT metabolism at $1 \mu \mathrm{M}$ (Fig. 2a). CrJ also significantly inhibited the PHT metabolism with $0.3 \%$ and $1 \%$ of the sample (Fig. $2 \mathrm{~b}$ ).

\section{Lineweaver-Burk plot for the inhibition of the PHT metabolism by $\mathrm{CrJ}$}

When same concentration of $\mathrm{CrJ}$ was added to microsome mixture with different PHT concentrations, the metabolizing ratio of PHT increased in a substrate-concentration-dependent manner. These two lines shown in Lineweaver-Burk plot crossed in the vicinity of Y-axis (the reciprocal of the metabolizing ratio) (Fig. 3 ).

\section{Influence of fluvoxamine on the metabolism of PHT by human liver microsomes}

To investigate whether $\mathrm{CrJ}$ inhibited the PHT metabolism directly by the suppression of CYP2C9

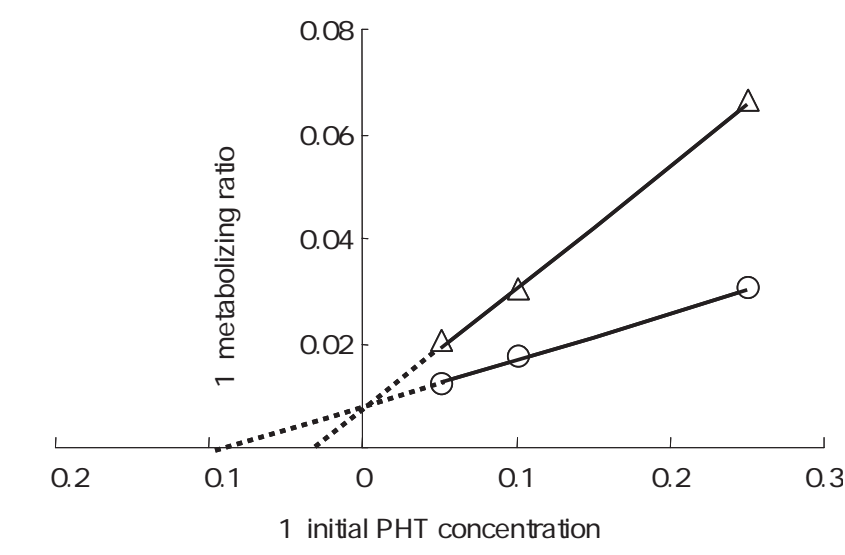

Fig. 3 Lineweaver-Burk plot for the influence of $\mathrm{CrJ}$ extract on the PHT metabolism

Lines were calculated by linear approximation. Initial PHT concentrations were 4,10 and $20 \mu \mathrm{g} / \mathrm{mL}$. $\bigcirc: 0.3 \%$ of $\mathrm{CrJ}$, $\triangle: 1.0 \%$ of $\mathrm{CrJ}$. $\mathrm{CrJ}$ concentration indicates the original concentration of $\mathrm{CrJ}$. Each plot shows the mean value from 3 experiments.

activity, fluvoxamine, the CYP1A2 and CYP2C19 inhibitor, was applied to this reaction. Fluvoxamine per se did not inhibit the PHT metabolism (Fig.4). However, $1 \%$ of $\mathrm{CrJ}$ plus $10 \mu \mathrm{M}$ fluvoxamine significantly decreased the PHT metabolism.

\section{Influence of $\mathrm{CrJ}$ on the PHT accumulation in 3D-HepG2 cells}

To investigate the penetration into hepatic cells of inhibitory substances in CrJ, HepG2 cells in 3dimension matrix (3D-HepG2) were used. The decreases in PHT concentration in media did not significantly differ among these groups (data not shown). PHT concentration in 3D-HepG2 cells in the SFZ group was significantly higher than that in the control group (Fig. 5). On the other hand, PHT concentration in the $\mathrm{CrJ}$ group was slightly increased, but it did not reach a statistical significance.

\section{Discussion}

In this study, we examined the inhibitory effect of CrJ extract on the CYP2C9 activity. First experiment using a baculovirus expression system showed that CrJ extract inhibited the metabolite production concentration-dependently. A previous report showed that $\mathrm{CrJ}$ had moderate inhibitory potential on flurbiprofen hydroxylation by human liver microsomes ${ }^{5}$, which is similar to the present data. The $\mathrm{IC}_{50}$ value of $\mathrm{CrJ}$ was approximately 3 -fold higher in their 


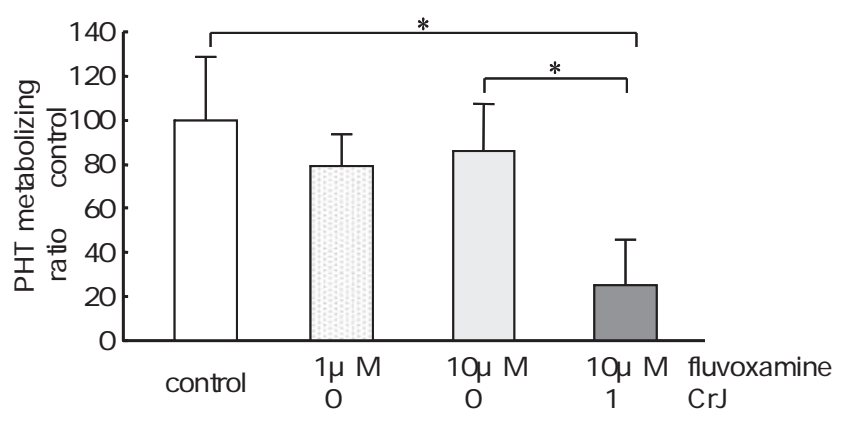

Fig. 4 Effect of fluvoxamine and $\mathrm{CrJ}$ extract on the metabolism of PHT by human liver microsomes

Initial concentration of PHT was $10 \mu \mathrm{g} / \mathrm{mL}$ and the incubation time was $40 \mathrm{~min}$. CrJ concentration indicates the original concentration of CrJ. ${ }^{*}, p<0.05 . \quad$ Mean \pm S.D. $\quad \mathrm{n}=3$.

report than in this study. However this parameter can not be directly compared between the different studies because the $\mathrm{IC}_{50}$ value depends on the substrate used and substrate concentration even if the same enzyme is inhibited.

In the second experiment, we examined an interaction between $\mathrm{CrJ}$ and PHT using human liver microsomes. SFZ, a positive control, significantly suppressed the PHT metabolism by $1.0 \mu \mathrm{M}$ and the $\mathrm{IC}_{50}$ value was estimated to be $0.3-1.0 \mu \mathrm{M}$. The $\mathrm{IC}_{50}$ value for $\mathrm{SFZ}$ on the CYP2C9 inhibition was reported in the range of $0.1-1.0 \mu \mathrm{M}^{11 \sim 13)}$, and therefore, we think that the present assay was valid. As to CrJ, its extract also concentration-dependently inhibited the PHT metabolism and the $\mathrm{IC}_{50}$ value was estimated to be $0.3-1.0 \%$, which is similar to that in the previous assay. In addition, to determine potential mechanism in the enzymatic-reaction inhibition of the PHT metabolism by CrJ, Lineweaver-Burk plot analysis was performed. The present finding indicates that the mode of the influence of $\mathrm{CrJ}$ on the PHT metabolism is a competitive inhibition. Thus, these findings support the hypothesis that $\mathrm{CrJ}$ inhibits the metabolism of CYP2C9 substrates.

Several biases were involved in the present experimental procedures. First, we used the organic concentrate extract of $\mathrm{CrJ}$. Using the concentrate extract, we can test each solvent at the same volume in each reaction mixture. However, this approach can not evaluate the potential effect of aqueous components of beverages. Second, because we used $\mathrm{TDx}^{\circledR}$ system of PHT which can not measure the metabolites, we measured the parent drug alone.

When human liver microsome assay was performed

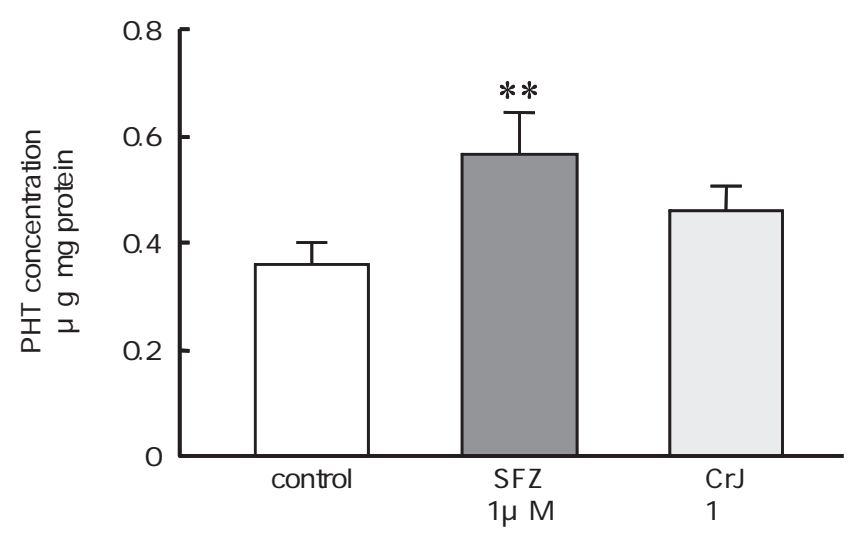

Fig. 5 Effect of SFZ and CrJ extract on the PHT concentrations in 3D-HepG2 cells

Cells were exposed to $20 \mu \mathrm{M}$ rifampicin for $48 \mathrm{hr}$ to induce CYP enzymes. Initial concentration of PHT in culture medium was $10 \mu \mathrm{g} / \mathrm{mL}$ and the incubation time was $40 \mathrm{~min}$. CrJ concentration indicates the original concentration of $\mathrm{CrJ}$.

${ }^{* *}, p<0.01$ compared with control. Mean \pm S.D. $\mathrm{n}=3$.

without NADPH-regeneration systems, PHT concentration was not changed from base-line level (data not shown). Therefore, it is unlikely that the inhibition of PHT metabolism by $\mathrm{CrJ}$ was via non-enzymatic reaction. PHT is reported to be mainly oxidized by CYP2C9 and partially by CYP2C198). To investigate whether CrJ especially inhibited CYP2C9 activity in PHT metabolism, we applied fluvoxamine, the CYP1A2 and CYP2C19 inhibitor ${ }^{14,15)}$, in this mircosome assay. Although fluvoxamine did not suppress the PHT metabolism, CrJ with fluvoxamine significantly decreased the PHT metabolism by about $30 \%$. Thus, we think that $\mathrm{CrJ}$ inhibited the PHT metabolism mainly through the suppression of CYP2C9 activity.

We did not determine the substance(s) which inhibited the PHT metabolism in this assay. Thus, it remains to be determined whether the substance(s) which decreases the PHT metabolism in the present assay has an efficient bioavailability to inhibit the CYP2C9 activity in vivo. To address this issue, we performed an experiment using 3D-HepG2 cells to examine the penetration of the inhibitory substance (s) into hepatic cells. SFZ significantly elevated the PHT concentration in cells without accelerating the PHT uptake, which might be due to the inhibition of CYP2C9 activity by SFZ. However, CrJ did not significantly increase the PHT concentration. Based on these findings, we think that the penetration of inhibitory substance (s) contained in $\mathrm{CrJ}$ into hepatic 
cells is small, and the inhibitory effect of $\mathrm{CrJ}$ is negligible in vivo, if any.

In summary, the present in vitro studies showed that $\mathrm{CrJ}$ extract inhibited the PHT metabolism by human liver microsomes. These data are comparable with the idea that CrJ inhibits the metabolism of drugs metabolized by CYP2C9. We did not perform a clinical trial in patients or healthy volunteers, because of the safety concern about PHT with a narrow therapeutic range ${ }^{16)}$. However, the present study suggests that the inhibitory effect of $\mathrm{CrJ}$ on the metabolism of CYP2C9 substrates is small in human subjects. Since in vivo study of a food-drug interaction is complicated by a number of factors, it seems difficult to extrapolate the in vitro data into in vivo. To determine whether the ingestion of $\mathrm{CrJ}$ causes the pharmacokinetic and pharmacodynamic alterations in human subjects, further clinical studies using CYP2C9 substrates other than warfarin or phenytoin are needed.

\section{Acknowledgement}

We thank Kanoko Egashira（Nagasaki University Hospital） and Yosuke Taniguchi (Kyushu University) for their valuable advice in the microsomal experiments. We also thank Tomohiro Maekawa（Jichi Medical University） for his experimental assistance.

\section{References}

1) Miners JO, Birkett DJ. Cytochrome P4502C9 : an enzyme of major importance in human drug metabolism. $\mathrm{Br} \mathrm{J}$ Clin Pharmacol $1998 ; 45: 525-38$.

2) Rettie AE, Jones JP. Clinical and toxicological relevance of CYP2C9 : drug-drug interactions and pharmacogenetics. Annu Rev Pharmacol Toxicol $2005 ; 45: 477-94$.

3) Suvarna R, Pirmohamed M, Henderson L. Possible interaction between warfarin and cranberry juice. BMJ $2003 ; 327$ : 1454 .

4) Rindone JP, Murphy TW. Warfarin-cranberry juice interaction resulting in profound hypoprothrombinemia and bleeding. $A m \mathrm{~J}$ Ther $2006 ; 13: 283-4$.

5) Lilja JJ, Backman JT, Neuvonen PJ. Effects of daily ingestion of cranberry juice on the pharmacokinetics of warfarin, tizanidine, and midazolam--probes of CYP2C9, CYP1A2, and CYP3A4. Clin Pharmacol Ther 2007 ; 81 : 833-9.

6) Ansell J, McDonough M, Harmatz JS, Greenblatt DJ. A randomized, double-blind trial of the interaction between cranberry juice and warfarin. J Thromb Thrombolysis 2008 ; 25 (1) : 112.

7) Greenblatt DJ, von Moltke LL, Perloff ES, Luo Y, Harmatz JS, Zinny MA. Interaction of flurbiprofen with cranberry juice, grape juice, tea, and fluconazole : in vitro and clinical studies. Clin Pharmacol Ther $2006 ; 79: 125-33$.

8) Bajpai M, Roskos LK, Shen DD, Levy RH. Roles of cytochrome P4502C9 and cytochrome P4502C19 in the stereoselective metabolism of phenytoin to its major metabolite. Drug Metab Dispos $1996 ; 24: 1401-3$.

9) Egashira K, Ohtani H, Itoh S, Koyabu N, Tsujimoto M, Murakami $\mathrm{H}$, et al. Inhibitory effects of pomelo on the metabolism of tacrolimus and the activities of CYP3A4 and P-glycoprotein. Drug Metab Dispos 2004 ; 32 : 828-33.

10) Koshimizu TA, Ueno S, Tanoue A, Yanagihara N, Stojilkovic SS, Tsujimoto G. Heteromultimerization modulates P2X receptor functions through participating extracellular and C-terminal subdomains. J Biol Chem 2002 ; 277 : 46891-9.

11) Baldwin SJ, Bloomer JC, Smith GJ, Ayrton AD, Clarke SE Chenery RJ. Ketoconazole and sulphaphenazole as the respective selective inhibitors of P4503A and 2C9. Xenobiotica 1995 ; $25: 261-70$.

12) Dierks EA, Stams KR, Lim HK, Cornelius G, Zhang H, Ball SE. A method for the simultaneous evaluation of the activities of seven major human drug-metabolizing cytochrome P450s using an in vitro cocktail of probe substrates and fast gradient liquid chromatography tandem mass spectrometry. Drug Metab Dispos $2001 ; 29: 23-9$

13) Lin T, Pan K, Mordenti J, Pan L. In vitro assessment of cytochrome P450 inhibition : strategies for increasing LC/MS based assay throughput using a one-point IC (50) method and multiplexing high-performance liquid chromatography. J Pharm Sci $2007 ; \mathbf{9 6}$ (9) : 2485-93.

14) Yamazaki H, Inoue K, Shaw PM, Checovich WJ, Guengerich FP, Shimada T. Different contributions of cytochrome P450 2C19 and $3 \mathrm{~A} 4$ in the oxidation of omeprazole by human liver microsomes : effects of contents of these two forms in individual human samples. J Pharmacol Exp Ther 1997 ; 283 : 434-42.

15) $\mathrm{Xu} \mathrm{ZH,} \mathrm{Xie} \mathrm{HG,} \mathrm{Zhou} \mathrm{HH.} \mathrm{In} \mathrm{vivo} \mathrm{inhibition} \mathrm{of} \mathrm{CYP2C19} \mathrm{but} \mathrm{not}$ CYP2D6 by fluvoxamine. Br J Clin Pharmacol $1996 ; 42: 518-21$.

16) Yukawa E. Optimisation of antiepileptic drug therapy. The importance of serum drug concentration monitoring. Clin Pharmacokinet $1996 ; 31:$ 120-30. 